\title{
Daily functioning of dyspnea, self-esteem and physical self in patients with moderate COPD before, during and after a first inpatient rehabilitation program
}

\author{
GREGORY NINOT ${ }^{1}$, GREGORY MOULLEC ${ }^{1}$, JACQUES DESPLAN ${ }^{2}$, \\ CHRISTIAN PREFAUT ${ }^{3} \&$ ALAIN VARRAY ${ }^{4}$ \\ 1 \\ University of Montpellier I, UFR STAPS, Laboratory ҰE 2416 "Engineering of Symbolic Processes for Sport and Health", \\ Montpellier, ${ }^{2}$ Clinique du Souffle La Solane, Osséja, ${ }^{3}$ UPRES-EA 701 Laboratory Physiology of Interactions, Faculty of \\ Medicine, University of Montpellier I, and ${ }^{4}$ University of Montpellier I, UFR STAPS, Laboratory EA 2991, Laboratory \\ Motor Efficiency and Deficiency, Montpellier, France
}

Accepted October 2006

\begin{abstract}
Purpose. Inpatient rehabilitation improves dyspnea and increases self-esteem between admission and discharge in patients with moderate chronic obstructive pulmonary disease (COPD). Some researchers nevertheless argue that the changes may be due to nursing effects and thus that scores will decrease quickly at home after discharge. This study assessed the change in dyspnea, self-esteem and physical self mean scores and stability in patients with moderate COPD during three consecutive four-week periods: at home, during an inpatient rehabilitation program, and again at home post-discharge.

Methods. Twenty-three consecutive patients [63.9 years (SD 6.6)] with moderate COPD [FEV $1=55.8 \%(S D 13.2)$ ] were included. The participants responded to the Physical Self Inventory and rated dyspnea using a visual analogue scale twice a day. Exercise tolerance was assessed with the six-minute walk test (6MWT) at admission and discharge.

Results. 6MWT performance improved between admission and discharge [452.3 m. (SD 74.0) vs. 503.3 m. (SD 80.4), $p<0.001$ ]. Dyspnea ratings improved $(p<0.001)$, as did the self-esteem and physical self scores between the two home periods $(p<0.001)$. The group showed less instability (SD and range) in their assessments of physical self-worth at home post-discharge compared to pre-admission $(p<0.01)$. Before rehabilitation, the correlation coefficients between dyspnea, and self-esteem, the perceptions of physical condition and attractive body were all significant. After rehabilitation, the coefficients between dyspnea, and perceived physical condition, physical strength and sport competence were significant $(p<0.05)$.

Conclusions. The results suggest that a first rehabilitation program increases the mean physical self scores in patients with moderate COPD and decreases their instability; the program also improves dyspnea. However, the impact of rehabilitation was greater on specific perceptions of physical abilities than on the global self-esteem. Randomized controlled trials are needed to confirm these changes, which were probably due to rehabilitation program.
\end{abstract}

Keywords: Daily functioning, dyspnea, rehabilitation, self-perception

\section{Introduction}

Randomized controlled trials have shown that multidisciplinary inpatient rehabilitation programs improve dyspnea scores and exercise tolerance between the day of admission and the day of discharge in patients with moderate chronic obstructive pulmonary disease (COPD) [1-3]. These programs also improve selfperceptions such as quality of life scores $[1,4,5]$ perceived competence to walk $[4,5]$, self-esteem $[6,7]$, and coping strategies $[8,9]$. None of the studies to date, however, has investigated the stability of these perceived dimensions at home before and after the rehabilitation program.

The low initial scores on admission may be due to the fatigue engendered by a long trip to the hospital or to anxieties about hospitalization. They may also be due to symptom exacerbation, such as increased

Correspondence: Gregory Ninot, Université Montpellier I, Faculté des Sciences du Sport et de 1'Education Physique, UFR STAPS, 700 avenue du Pic St-Loup, 34090 Montpellier, France. Tel: +33467 415 731. Fax: +33467415 708. E-mail: gregory.ninot@univ-montp1.fr 
dyspnea, wheezing, cough, sputum production, and worsened gas exchange [10]. Indeed, many factors interfere with the maintenance of stable selfevaluative perceptions, and patients with COPD have been particularly noted to underestimate their questionnaire ratings [7].

Studies based on a daily assessment protocol have repeated the measures several months after rehabilitation. No clear consensus has emerged from these studies [5]. The data suggest either a progressive or sudden decrease in the self-perceptions in patients at six months [11], one year [12] or two years [4]. After an inpatient rehabilitation program, the high scores on discharge day may be overestimations designed to "thank" the rehabilitation team and, if this is the case, they are destined to quickly decrease with the end of nursing care. Few days after the end of a rehabilitation program, the decrease may be due to internal factors such as highly fatigue due to degree of difficulty of exercise, exacerbation, age, anxiety, lack of motivation (being too busy or lacking interest), personal belief (be reminders of the irreversibility of respiratory disease), ineffective adherence to medications and health-care activities, or to external factors such as setting and social support.

Recent research has emphasized an important characteristic of self-perception: Instability $[13,14]$. For example, individuals with unstable self-esteem show more extreme emotional and behavioral reactions to events involving threats to self-esteem than people with stable self-esteem. These individuals also experience anger and hostility more often [15]. The instability of self-esteem reveals central information about depression $[15,16]$ and quality of life changes [17]. An approach that has been used effectively in research investigating stability in self-perception is dynamical psychology [14]. The theoretical approach, methodological principles and psychometric instruments adapted to time series need to be appropriate. The theoretical approach focuses not only on self-perception scores as in classic research (mean score), but also on intra-individual instability (standard deviation or range). Self-esteem is considered as a complex system subjected to several constraints over time, and time series analysis is an appropriate and highly informative means to go beyond the limits of inter-individual studies in order to extract new data on intra-individual instability. Dynamical social psychology requires standardized measures to construct time series [14]. Since the repeated measures need to be numerous and regular, short inventories are best suited to assess day-to-day self-perceptions. The instruments need to be easy to use, sensitive, reliable and valid $[18,19]$.

To our knowledge, no researcher has examined the instability of self-perceptions in patients with COPD over a rehabilitation period. The stability in perceived physical abilities, global self-esteem and dyspnea during the post-rehabilitation period in comparison with the pre-rehabilitation period could indicate a significant change over time demonstrating the abilities to cope with effort constraints. The present study assessed the day-to-day instability of dyspnea, global self-esteem and physical condition in patients with moderate COPD over three months, including during a four-week rehabilitation program in the middle of the study period. The purpose was to examine whether inpatient rehabilitation programs not only increase of self-perception scores but also reduce their instability over time.

\section{Methods}

\section{Patients}

Twenty-three consecutive patients with moderate COPD took part in this study $(63.9 \pm 6.6$ years $)$. Patients were included in the study if their forced expiratory volume in one second $\left(\mathrm{FEV}_{1}\right)$, measured at a time of clinical stability, was less than $60 \%$ of the predicted value with less than $20 \%$ reversibility in response to a $\beta$-agonist $\left(\mathrm{FEV}_{1}=55.8 \pm 13.2 \%\right.$, $\left.\mathrm{FEV}_{1} / \mathrm{FVC}=64.8 \pm 17.4 \%\right)$. A patient was defined as having stable moderate disease if he or she had had no change in symptoms or medication for two months before entry. Patients were for the most part ex-smokers $(75 \%)$. Most were not working (83\%) due to long-term disease or retirement. The marital status was $80 \%$ married or living together, $10 \%$ divorced, and 10\% single.

Patients were excluded if they were oxygendependent or if they had a pharmacologically treated psychiatric disorder that would affect psychological function. The protocol was approved by the institutional review board of the rehabilitation center and all patients gave informed written consent to participate.

\section{Study design}

All patients were participating for the first time in an inpatient rehabilitation program based on individualized exercise training. Each of them completed a brief questionnaire on a single page of a personal week notepad twice a day between 7:00 and 9:00 (a.m. and p.m.) over three four-week periods: Before, during, and after the rehabilitation program. The participants handed into the researcher at the end of four week periods (rehabilitation admission and discharge) and mailed the last four notepad. Each page of the week notepad presented seven randomized items: Six items from the Physical Self Inventory-6 (PSI6) [18], and one for perceived dyspnea. The participants based their responses on how they felt at the moment they were completing 
each form. They assessed their immediate feelings of self-perception. Therefore, we collected 168 consecutive observations per scale for the individual time series. We did not use method to reduce the missing data. If a period had more than $5 \%$ of missing data, we planned to exclude the participant (cut-off point). Nevertheless, the compliance of the patients to the short questionnaire was entire (from 4.1 to $0 \%$ of missing data). Three patients refused to participate in the study. The analysis of the individual time series was appropriate to determine the individual resistance to environmental stimuli per period [16].

\section{Rehabilitation program}

The patients participated in the rehabilitation program five days per week in small groups of eight to ten. The program lasted for four successive weeks and included the following: (a) individualized exercise training (4 hours per day) [20]; (b) respiratory therapy with aerosol and/or drainage ( $1 \mathrm{~h}$ per day); (c) health education ( $2 \mathrm{~h}$ per week) with information on pulmonary disease, medications, and healthful daily living strategies; (d) psychosocial support (discussion group $1 \mathrm{~h}$ per week); and (e) dietary monitoring (1 h per week). The training included cycling, walking, general exercises, and relaxation. The cycling component consisted of $45 \mathrm{~min}$ on a cycle ergometer with three successive pedaling periods of $10 \mathrm{~min}$ separated by $5 \mathrm{~min}$ of active recovery. The exercise intensity was individualized and determined by the heart rate corresponding to the ventilatory threshold (Vth) assessed during an incremental exercise test. After cycling, the patients stretched for $10 \mathrm{~min}$ and then relaxed for $30 \mathrm{~min}$. The second component was $2 \mathrm{~h}$ of nature walking with $45 \mathrm{~min}$ at Vth intensity and $1.15 \mathrm{~h}$ at lower walking speed. Cycling, general exercises, and relaxation were done in the same half-day, and walking was done in the other half-day.

\section{Measurement instruments}

Self-esteem and physical self. The PSI6, which has been validated in French, was used to assess global self-esteem and physical self [18]. This instrument is a six-item questionnaire scored with a visual analogue scale: Under each item is a single $10-\mathrm{cm}$ horizontal line without formal indications that can be scored from "not at all" (marked at $0.0 \mathrm{~cm}$ ) to "absolutely" (marked at $10.0 \mathrm{~cm}$ ). A single-item measure eliminates item redundancy and therefore reduces fatigue, frustration, and boredom associated with answering highly similar item repeatedly [19]. This instrument measures six hierarchically organized dimensions $[18,21]$. The apex of the hierarchy is occupied by global self-esteem, the feeling that everyone has about his or her own value. The median level is occupied by physical self-worth, which reflects general feelings of satisfaction and confidence about the physical domain of self. Physical self-worth is then decomposed into four subdomains. Physical condition reflects the evaluation of physical condition. Sport competence corresponds to perceptions about sport and athletic ability. Physical strength represents perceived strength and muscle development. Attractive body is related to perceived appearance. The PSI6 is specifically designed to allow repeated administration and reveal stability in self-esteem and physical self. This instrument was proven to reproduce the hierarchical structure of the corresponding multi-item inventories using correlations and partial correlations, factor analysis, and confirmatory factor analysis $[21,22]$, as well as for its external validity [21].

Dyspnea. One specific item about dyspnea was added to each inventory in order to measure current dyspnea. The patient thus made a vertical mark on the corresponding horizontal line anywhere from 0.0 for "no breathlessness" to $10.0 \mathrm{~cm}$ for "complete breathlessness".

Exercise tolerance. The exercise tolerance of each patient was assessed by the six-minute walk test (6MWT) on the admission and discharge days. Because of the documented influence of test familiarization, a $6 \mathrm{MWT}$ protocol comprising two repetitions was chosen to provide relatively reproducible results $[12,20]$. Each patient performed the 6MWT in an empty, level, 20-m hospital corridor. Each 6MWT was supervised by a physical therapist who provided standardized encouragement but did not walk alongside the patient. Before the first test, the patient's pulse and respiratory rate were measured to establish baseline values; they were measured again before the second test to verify that the values had returned to baseline. The greater distance covered in the two $6 \mathrm{MWT}$ s was retained for data analysis.

\section{Statistical analysis}

Data are reported as the group means of the individual time series from each period for each subjective dimension \pm standard deviation (SD). One-way analyses of variance (ANOVA) for repeated measures were employed to assess changes between means, standard deviations, and ranges across the four-week periods (before, during, and after rehabilitation). All variables were tested for normality with the Shapiro-Wilk test.

For home pre-admission and home post-discharge periods, the Pearson correlation test was conducted between the time series for the self-perception 
dimensions and dyspnea. Paired Student's $t$-tests were used to compare $6 \mathrm{MWT}$ performance between admission and discharge. A $p$ value of $<0.05$ was considered significant for these analyses.

\section{Results}

\section{Exercise tolerance and dyspnea}

Patients showed significantly improved 6MWT performance at discharge $(452.3 \pm 74.0 \mathrm{~m}$ on admission vs. $503.3 \pm 80.4 \mathrm{~m}$ at discharge, $p<0.001$ ). Table I shows that the dyspnea scores changed significantly over the three periods $[\mathrm{F}(2,44)=9.17$; $p<0.001]$. The score significantly decreased between the two home periods.

Concerning dyspnea instability, the ANOVA did not show a significant effect for standard deviation $[\mathrm{F}(2,44)=1.4 ; p=0.25]$ (see Table II), though it revealed a significant effect for range $[\mathrm{F}(2,44)=3.4$; $p<0.05$ ] (see Table III). The results based on comparison between the group means of the individual ranges over the three periods showed a significant decrease in dyspnea between the inpatient rehabilitation period and the home discharge period.

At home before rehabilitation, significant correlations were obtained between the time series for dyspnea and for global self-esteem $(r=-0.50$, $p=0.01)$, attractive body $(\mathrm{r}=-0.48, p=0.01)$ and physical condition $(r=-0.49, p=0.01)$. At home after rehabilitation, significant correlations were found between the time series for dyspnea and for physical condition $(r=-0.48, p=0.01)$, physical strength $(r=-0.45, p=0.01)$ and sport competence $(r=-0.47, p=0.01)$. Figure 1 illustrates the findings of individual changes in dyspnea and perceived physical condition.

\section{Level of self-perceptions}

The ANOVA performed on the PSI6 scales revealed a significant effect on time: global self-esteem $[\mathrm{F}(2,44)=15.8 ; p<0.001]$, physical self-worth $[\mathrm{F}(2,44)=30.2 ; \quad p<0.001]$, physical condition $[\mathrm{F}(2,44)=26.1 ; p<0.001]$, sport competence $[\mathrm{F}(2,44)=39.1 ; p<0.001]$, attractive body $[\mathrm{F}(2,44)=11.7 ; p<0.001]$, and physical strength $[\mathrm{F}(2,44)=27.1 ; p<0.001]$. Each dimension measured by the PSI6 showed a significant increase in the mean score between the before and after periods of inpatient rehabilitation, and between before rehabilitation and during rehabilitation (Table I). The sub-domains related to perceived physical abilities (physical condition, sport competence, and physical strength), physical self-worth, and global self-esteem increased between the rehabilitation period and the post-discharge home period.

Table I. Change in mean score of self-perceptions before, during, and after an inpatient rehabilitation program in patients with moderate COPD $(n=23)$.

\begin{tabular}{|c|c|c|c|c|c|c|}
\hline & Home & Rehabilitation & Home & $\mathrm{P} 1<\mathrm{P} 2$ & $\mathrm{P} 2<\mathrm{P} 3$ & $\mathrm{P} 1<\mathrm{P} 3$ \\
\hline Global self-esteem & $4.3(2.1)$ & $5.2(1.8)$ & $6.0(1.8)$ & $*$ & $*$ & $* * *$ \\
\hline Physical self-worth & $3.6(1.7)$ & $4.6(1.3)$ & $5.7(1.5)$ & $* *$ & $* *$ & $* * *$ \\
\hline Physical condition & $3.2(1.6)$ & $4.6(1.3)$ & $5.5(1.6)$ & $* * *$ & * & $* * *$ \\
\hline Sport competence & $3.0(1.8)$ & $4.5(1.5)$ & $5.5(1.6)$ & $* * *$ & $* *$ & $* * *$ \\
\hline Attractive body & $4.0(2.3)$ & $4.6(2.2)$ & $5.0(2.6)$ & $*$ & NS & $* * *$ \\
\hline Physical strength & $3.2(1.7)$ & $4.4(1.4)$ & $5.5(1.6)$ & $* * *$ & $* *$ & $* * *$ \\
\hline Dyspnea & $5.6(2.0)$ & $4.8(1.3)$ & $3.7(1.5)$ & NS & NS & $* * *$ \\
\hline
\end{tabular}

Data are means (SD). NS, non-significant; ${ }^{*} p<0.05 ;{ }^{* *} p<0.01 ; * * p<0.001$ (obtained with the Scheffé test); P1, 4-week home period, pre-rehabilitation; P2, 4-week period of inpatient rehabilitation; P3, 4-week home period, post-rehabilitation.

Table II. Change in standard deviation of self-perceptions before, during, and after an inpatient rehabilitation programs in patients with moderate COPD $(n=23)$.

\begin{tabular}{|c|c|c|c|c|c|c|}
\hline & Home & Rehabilitation & Home & $\mathrm{P} 1<\mathrm{P} 2$ & $\mathrm{P} 2<\mathrm{P} 3$ & $\mathrm{P} 1<\mathrm{P} 3$ \\
\hline Global self-esteem & $0.95(0.58)$ & $1.12(0.76)$ & $0.70(0.27)$ & NS & $* *$ & NS \\
\hline Physical self-worth & $1.04(0.51)$ & $1.17(0.74)$ & $0.70(0.35)$ & NS & $* * *$ & $*$ \\
\hline Physical condition & $0.97(0.63)$ & $1.26(0.70)$ & $0.75(0.40)$ & NS & $* * *$ & NS \\
\hline Sport competence & $0.92(0.54)$ & $1.29(0.76)$ & $0.69(0.35)$ & $*$ & $* * *$ & NS \\
\hline Attractive body & $0.85(0.53)$ & $0.99(0.76)$ & $0.54(0.31)$ & NS & $* *$ & NS \\
\hline Physical strength & $0.94(0.53)$ & $1.24(0.74)$ & $0.72(0.35)$ & NS & $* * *$ & NS \\
\hline Dyspnea & $1.05(0.67)$ & $1.21(0.74)$ & $0.98(0.63)$ & NS & NS & NS \\
\hline
\end{tabular}

Data are means (SD). NS, non-significant; * $p<0.05$; ** $p<0.01{ }^{* * *} p<0.001$ (obtained with the Scheffé test); P1, 4-week home period, pre-rehabilitation; P2, 4-week period of inpatient rehabilitation; P3, 4-week period, post-rehabilitation. 
Table III. Change in range of self-perceptions before, during, and after an inpatient rehabilitation program in patients with moderate COPD $(n=23)$.

\begin{tabular}{lcccccc}
\hline & Home & Rehabilitation & Home & P1 $<$ P2 & P2 $<$ P3 & P1 $<$ P3 \\
\hline Global self-esteem & $3.8(1.9)$ & $4.2(2.2)$ & $3.2(1.2)$ & NS & NS & NS \\
Physical self-worth & $4.4(1.8)$ & $4.5(2.2)$ & $3.2(1.7)$ & NS & $* *$ & NS \\
Physical condition & $4.0(2.4)$ & $5.0(2.4)$ & $3.3(2.0)$ & NS & $* *$ & NS \\
Sport competence & $4.1(2.1)$ & $4.6(2.3)$ & $3.0(1.5)$ & NS & NS & $* *$ \\
Attractive body & $4.1(2.3)$ & $3.2(2.5)$ & $2.3(1.1)$ & NS & NS & $*$ \\
Physical strength & $3.9(1.9)$ & $5.0(2.6)$ & $3.3(1.5)$ & $3.6(1.9)$ & NS \\
Dyspnea & $4.5(2.6)$ & $5.1(2.7)$ & NS \\
\hline
\end{tabular}

Data are means (SD). NS, non-significant; ${ }^{*} p<0.05 ;{ }^{* *} p<0.01 ; * * p<0.001$ (obtained with the Scheffé test); P1, 4-week home period, pre-rehabilitation; P2, 4-week period of inpatient rehabilitation; P3, 4-week period, post-rehabilitation.

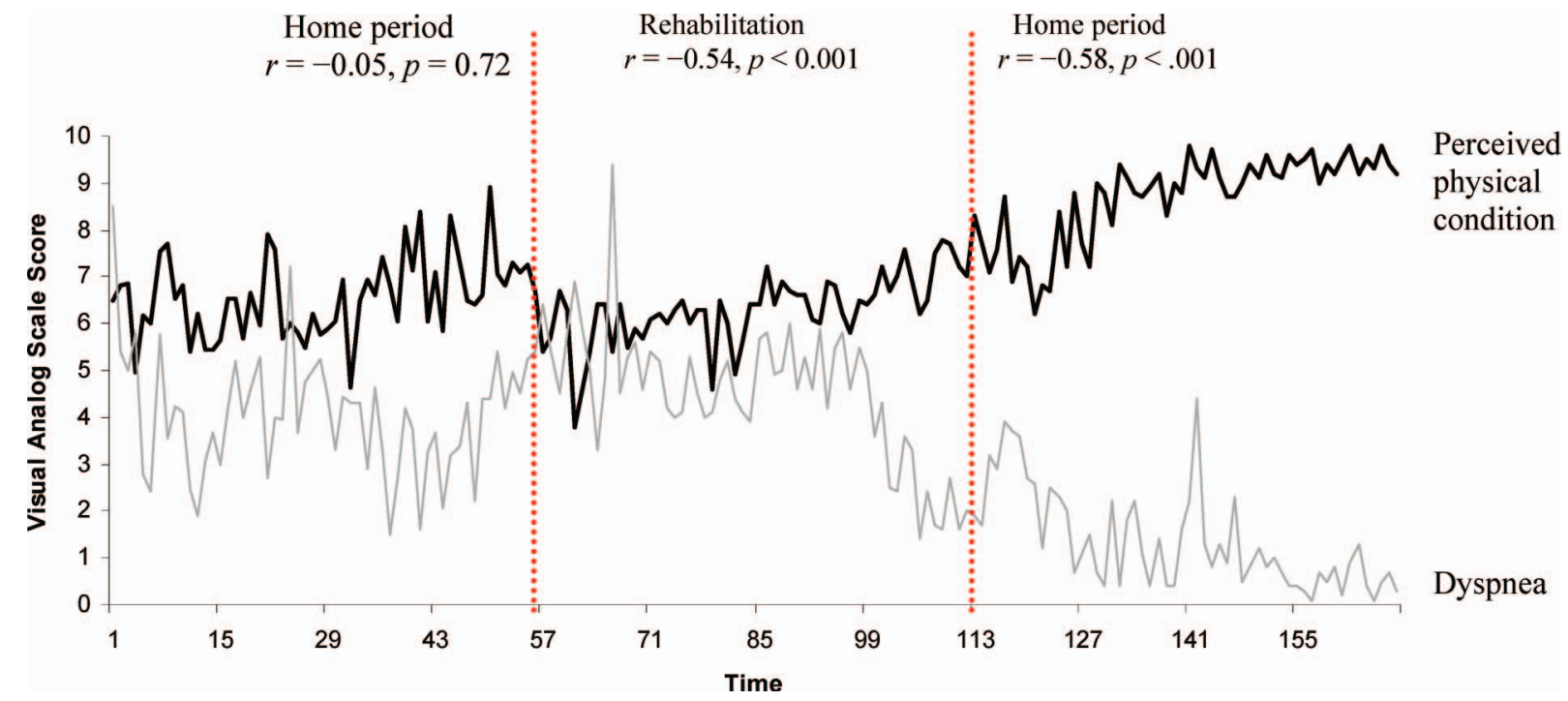

Figure 1. Dynamic change in dyspnea and perceived physical condition in a COPD patient with moderate COPD over 3 consecutive periods of 4 weeks (home, inpatient rehabilitation program, and home). The patient scored twice a day, between 7:00 and 9:00 (a.m.) and between 7:00 and 9:00 (p.m.) on a 10-cm visual analogue scale.

\section{Instability of self-perception}

The ANOVA performed on the standard deviations of the PSI6 time series revealed a significant effect on time: Global self-esteem $[\mathrm{F}(2,44)=5.4 ; p<0.01]$, physical self-worth $[\mathrm{F}(2,44)=9.5 ; p<0.001]$, physical condition $[\mathrm{F}(2,44)=8.9 ; p<0.001]$, sport competence $[\mathrm{F}(2,44)=10.4 ; p<0.001]$, attractive body $[\mathrm{F}(2,44)=8.8 ; \quad p<0.001]$, and physical strength $[\mathrm{F}(2,44)=9.0 ; p<0.001]$. The results based on comparison between the group means of the individual standard deviations over the three periods showed a significant decrease in sub-domain scores, physical self-worth, and global self-esteem between the inpatient rehabilitation period and the home post-discharge period (Table II). Decreased instability was also observed between the two home periods for physical self-worth. Only instability in the perception of sport competence significantly increased between the home period before rehabilitation and the rehabilitation program.
The ANOVA performed on the range of the time series revealed a significant effect on time: physical self-worth $[\mathrm{F}(2,44)=7.5 ; p<0.01]$, physical condition $[\mathrm{F}(2,44)=4.7 ; p<0.01]$, sport competence $[\mathrm{F}(2,44)=6.9 ; \quad p<0.01]$, attractive body $[\mathrm{F}(2,44)=10.8 ; p<0.001]$, and physical strength $[\mathrm{F}(2,44)=6.0 ; p<0.01]$. The ANOVA showed a significant effect of dyspnea $[\mathrm{F}(2,44)=3.4 ; p<0.05]$. Sub-domain scores related to physical abilities, physical self-worth, and dyspnea showed significant decreases between the inpatient rehabilitation period and the home discharge period (Table III). Decreased instability was also observed between the two home periods for physical self-worth and attractive body.

\section{Discussion}

Patients with moderate COPD participating in their first inpatient rehabilitation program showed significantly decreased instability in specific perceived 
dimensions, as well as increases in these scores during the first month post-discharge.

During the month at home before the program, the inventory results agreed with previous studies $[7,9]$ demonstrating that COPD patients suffer from low global self-esteem (scores in the bottom half of the scale) $[18,24]$ compared to healthy population (mean level $=7.22, \mathrm{SD}=0.70$, minimal mean level $=5.18$, maximal mean level $=9.11)[18,25]$. Individuals with low self-esteem report more negative emotions [26] and greater sensitivity to negative events [16]. Moreover, the results highlighted the acute individual instability of self-esteem and the physical self dimensions compared to sedentary adults (mean of SD whatever the dimension $=0.60, \mathrm{SD}=0.70$, minimal mean of SD whatever the dimension $=0.29$, maximal mean of SD whatever the dimension $=0.82)[18,25]$. This instability could reflect a certain vulnerability to the impact of daily events. People with unstable self-esteem show more extreme emotional and behavioural reactions to events involving threats to self-esteem. For example, people with unstable self-esteem experience more anger and hostility than others do [15]. Both affect physical self-perceptions (except attractive body) and suggest a depressive episode [16,17]. In COPD patients never having participated in a rehabilitation program, the literature reveals that $42 \%[27,28]$ present a clinical depression compared to $5-10 \%$ of a normal population [29]. The weak and changeable self-perceptions may have three origins: (a) Chronicity may be a cause, as for example in patients who have been coping with COPD for at least 10 years. The decreased general functioning and the limitation of physical abilities may influence the accuracy of self-evaluations, with patients under- or overestimating their physical abilities in a random manner; (b) Fear of dyspnea, anxiety, and passive coping strategies [30] encourage the loss of a sense of self. Patients with COPD adopt emotional and avoidance coping strategies preferentially to active coping [31]. They lay emphasis on decreasing precipitate situations and avoid uncontrollable activities [32]. They try to calculate and plan every behavior in order to act with minimal effort and to control their setting [28]; (c) Lastly, these patients are restricted in many ordinary life functions (work, finances, leisure, and so on) and are more vulnerable to social isolation [33]. The consequences of weak and fluctuating perceptions of their physical abilities include greater risks of depressive and sedentary behavior, as well as greater risks of cardiovascular disease, social dependence, worsened quality of life, abuse of medication, and hospital readmission [34]. Further studies are needed to explore these assumptions.

This study based on intra-individual observations suggested that a first-time inpatient rehabilitation program improved dyspnea and increased global self-esteem and physical self scores in patients with moderate COPD. The findings support previous research indicating the beneficial effects of rehabilitation on dyspnea $[1,3]$, perceived walking abilities $[4,5]$, and global self-esteem $[6,7]$.

The rehabilitation program was reality-based, and it placed emphasis on residual physical abilities and not just respiratory disabilities. Attention was focused on improving exercise tolerance, desensitization to dyspnea and ways to limit reliance on avoidance coping strategies [31]. The high correlation between exercise tolerance and the physical condition sub-domain indicated that an improvement in exercise tolerance and dyspnea reinforces the corresponding sub-domain (physical condition), and then enhances physical self-worth level, and finally global self-esteem.

The most original finding of this study was the significantly greater stability in physical self-worth during the post-rehabilitation period in comparison with the pre-rehabilitation period. This change indicates a desire to maintain a positive and stable perception of one's physical competence, and thus, a prime motivation for controlling shortness of breath [7] and exercising regularly [21]. Multidisciplinary rehabilitation programs are founded on methodic principles. Participants with moderate COPD are taught to limit emotional overreactions and to try to understand and control every stressor (such as dyspnea) by using active coping. A first rehabilitation program functions as a constraint that begins by challenging existing perceptions of physical self and then proceeds by rebuilding these perceptions from a more accurate viewpoint. Nevertheless, the instability of physical self-subdomains did not decrease significantly between the two home periods (expect attractive body for range). This result can indicate difficulties to distinguish improvements on physical abilities (strength, endurance and motor skills) during only one rehabilitation session.

Global self-esteem, however, although it increased between the two home periods, remained unstable on a day-to-day basis. This may indicate that a depressive status will not change in long term, as reported in the literature $[2,4,7]$. A first-time inpatient rehabilitation program notably improves perceptions of physical self-worth, but if exercise is not continued at home or if exacerbations reoccur, self-esteem will automatically decrease. We can assume that the first rehabilitation program massively influences the cognitive part of self-perceptions, particularly those related to the concrete aspects of the self such as the physical dimensions [23]. In contrast, global perceptions are more closely related to emotions and thus provoke active resistance to change. 
For rehabilitation specialists, the study indicated that a first-time inpatient rehabilitation program had effects on perceptions in the concrete domain of physical self-worth, and that the effects lasted at least one month post-discharge. Improved perceptions of one's physical abilities and greater stability in physical self-worth should play a major role in reducing the severity of COPD symptoms, as earlier research has shown that positive and stable perceptions of one's physical abilities is the prime motivating factor for controlling shortness of breath and continuing regular exercise at home [7]. Sessions that desensitize patients to their fear of dyspnea, teach specific exercises, and provide health education and psychosocial group support respectively help them to control effort, improve exercise tolerance, and understand disease functioning and physical limits. The limits to reduce the instability of selfesteem and physical subdomains scores suggest that patients will fail to recall elements of potentially important medical advice and that a second rehabilitation program could be developed to focus more specifically on stabilizing self-esteem and distinguishing physical abilities. This program will be focus on change of status of patient with non acute disease but chronic disease and on maintain effects following rehabilitation with self-management practice and adherence to treatment protocol after discharge.

To conclude, a first program of inpatient rehabilitation increased global self-esteem and perceptions of physical abilities and stabilized physical self-worth in patients with mild to moderate COPD. Randomized controlled trials are needed to confirm these changes. Longitudinal investigations will be useful to determine the programs that best develop the stability of concrete self-perceptions and promote adherence to rehabilitation program [35].

\section{Acknowledgements}

The present study was conducted jointly with the "Clinique La Solane". The authors wish to thank all the staff of this establishment, particularly Audrey Brun, François Ceugnet, Monique Chambouleyron, Philippe Desnot, Sophie Leymarie, Bernard Palomba, Magali Partyka, and Magali Poulain for their positive and perspicacious contributions.

\section{References}

1. Goldstein RS, Gort EH, Stubbing D, et al. Randomised controlled trial of respiratory rehabilitation. Lancet 1994; 19:1394-1397.

2. Toshima MT, Blumberg E, Ries A, et al. Does rehabilitation reduce depression in patients with chronic obstructive pulmonary disease? J Cardiopulmonary Rehabil 1992;12: $261-269$.
3. Whiters NJ, Rudkin ST, White RJ. Anxiety and depression in severe chronic obstructive pulmonary disease: The effects of pulmonary rehabilitation. J Cardiopulmonary Rehabil 1999;19:362-365.

4. Ninot G, Fortes $M$, Leymarie S, Brun A, Poulain $M$, Desplan J, Varry A. Effects of an intensive period inpatient rehabilitation program on the perceived physical self in moderate COPD patients. Intl J Rehab Res 2002;25: $51-55$.

5. Lacasse Y, Wong E, Guyatt GH, et al. Meta-analysis of respiratory rehabilitation in chronic obstructive pulmonary disease. Lancet 1996;348:1115-1119.

6. Ketelaars CA, Abu-Saad HH, Schlösser MA, et al. Long-term outcome of pulmonary rehabilitation in patients with COPD. Chest 1997;112:363-369.

7. Kersten L. Changes in self-concept during pulmonary rehabilitation, part 1. Heart Lung 1990;19:456-462.

8. Ketelaars CA, Abu-Saad HH, Schlösser MA, Mostert R, Wouters EF. Long-term outcome of pulmonary rehabilitation in patients with COPD. Chest 1997;112:363-369.

9. Wempe JB, Wijkstra PJ. The influence of rehabilitation on behaviour modification in COPD. Patient Educ Counseling 2004;52:237-241.

10. Madison JM, Irwin RS. Chronic obstructive pulmonary disease. Lancet 1998;352:467-473.

11. Buchi S, Villiger B, Sensky T, Schwarz F, Wolf C, Buddeberg C. Psychosocial predictors of long-term success of in-patient pulmonary rehabilitation of patients with COPD. Eur Respiratory J 1997;10:1272-1277.

12. Griffiths TL, Phillips CJ, Davies S, Burr ML, Burr ML, Campbell IA. Cost effectiveness of an outpatient multidisciplinary pulmonary rehabilitation programme: A randomised controlled trial. Thorax 2001;56:779-784.

13. Nezlek JB, Plesko RM. Day-to-day relationships among selfconcept clarity, self-esteem, daily events, and moods. Personality Soc Psychol Bull 2001;27:201-211.

14. Nowak A, Vallacher RR. Dynamical social psychology. New York: The Guilford Press; 1998.

15. Kernis MH, Grannemann BD, Barclay LC. Stability and level of self-esteem as predictors of anger arousal and hostility. J Personality Soc Psychol 1989;56:1013-1022.

16. Greenier KD, Kernis $\mathrm{MH}$, McNamara $\mathrm{CW}$, et al. Individual differences in reactivity to daily events: Examining the roles of stability and level of self-esteem. J Personality 1999;67:185-208.

17. Barge-Schaapveld DQ, Nicolson NA, Berkhof J, et al. Quality of life in depression: Daily life determinants and variability. Psychiatric Res 1999;88:173-189.

18. Ninot G, Fortes $M$, Delignières D. A psychometric tool for the assessment of the dynamics of the physical self. Eur J Appl Psychol 2001;51:205-216.

19. Robins RW, Hendin HM, Trzesniewski KH. Measuring global self-esteem: Construct validation of a single-item measure and the Rosenberg self-esteem scale. Personality Soc Psychol Bull 2001;27:151-161.

20. Varray A, Préfaut C. Exercise training in patients with respiratory disease: Procedure and results. Eur Respiratory Rev 1995;5:51-58.

21. Fox KR, Corbin CB. The Physical Self Perception Profile: Development and preliminary validation. J Sport Exercise Psychol 1989;11:408-430.

22. Ninot G, Delignières $D$, Fortes $M$. L'évaluation de l'estime de soi dans le domaine corporel [Self-esteem assessment in the physical domain] STAPS 2000;51:35-48.

23. Griffiths TL, Burr ML, Campbell IA, et al. Results at 1 year of outpatient multidisciplinary pulmonary rehabilitation: A randomised controlled trial. Lancet 2000;335:362368. 
24. Baumeister RF. The nature and structure of the self: An overview. In: Baumeister RF, editor. The self in social psychology. Philadelphia: Taylor and Francis; 1999. pp 1-20.

25. Ninot G, Fortes $M$, Delignières D, Maïano C. The dynamic adjustment of physical self in adults over time. Indiv Differences Res 2004;2(2):137-151.

26. Strauman TJ, Higgins ET. Self-discrepancies as predictors of vulnerability to distinct syndromes of chronic emotional distress. J Personality 1988;56:685-707.

27. McSweeny AJ, Grant I, Heaton RK, et al. Life quality of patients with chronic obstructive pulmonary disease. Arch Internal Med 1982;142:473-148.

28. Light RW, Merrill EJ, Despars JA, et al. Prevalence of depression and anxiety in patients with COPD: Relationship to functional capacity. Chest 1985;87:35-38.

29. Weismann MM, Klerman GL. Depression: Current understanding and changing trends. Ann Rev Public Health 1992;13:319-339.

30. Scharloo M, Kaptein AA, Weinman J, et al. Illness perceptions, coping and functioning in patients with rheumatoid arthritis, chronic obstructive pulmonary disease and psoriasis. J Psychosomatic Res 1998;44:573-585.
31. Narsavage GL, Weaver TE. Physiologic status, coping, and hardiness as predictors of outcomes in chronic obstructive pulmonary disease. Nursing Res 1994;43:90-94.

32. Folgering H, Rooyackers J. Pulmonary rehabilitation in chronic obstructive pulmonary disease. Eur Respiratory J 1998;11:520-523.

33. Dudley DL, Glaser EM, Jorgenson BN, et al. Psychosocial concomitants to rehabilitation in chronic obstructive pulmonary disease. Part I. Psychosocial and psychological considerations. Chest 1980;77:413-420.

34. Rutten-van Molken MP, Postma MJ, Joore MA, et al. Current and future medical cost of asthma and chronic obstructive pulmonary disease in the Netherlands. Respiratory Med 1999;93:779-787.

35. Brooks D, Krip B, Mangovski-Alzamora S, et al. The effect of postrehabilitation programmes among individuals with chronic obstructive pulmonary disease. Eur Respiratory J 2002;20:20 - 29 . 